\title{
Identifying Galactic PeVatrons with Neutrinos
}

\author{
M.C. Gonzalez-Garcia* \\ C.N. Yang Institute for Theoretical Physics, \\ SUNY at Stony Brook, Stony Brook, NY 11794-3840, USA \\ Institució Catalana de Recerca $i$ Estudis Avançats (ICREA), \\ Departament d'Estructura i Constituents de la Matèria, \\ Universitat de Barcelona, 647 Diagonal, E-08028 Barcelona, Spain \\ Francis Halzen $\dagger$ \\ Department of Physics, University of Wisconsin, Madison, WI 53706, USA \\ Soumya Mohapatrat \\ C.N. Yang Institute for Theoretical Physics, \\ SUNY at Stony Brook, Stony Brook, NY 11794-3840, USA
}

\begin{abstract}
We perform a realistic evaluation of the potential of IceCube, a kilometer-scale neutrino detector under construction at the South Pole, to detect neutrinos in the direction of the potential accelerators of the Galactic cosmic rays. We take fully account of the fact that the measurement of the energy of the secondary muons can be used to further discriminate between the signal and the background of atmospheric neutrinos. We conclude that IceCube could identify the sources in the Milagro sky map as the sources of the Galactic cosmic rays at the $3 \sigma$ level in one year and at the $5 \sigma$ level in three years. We discuss the dependence of these expectations on ambiguities, mostly associated with our incomplete knowledge of the astrophysics of the sources.
\end{abstract}

PACS numbers: 95.55.Vj; 95.85.Pw; 95.85.Ry; 98.70.Sa

\footnotetext{
*Electronic address: concha@insti.physics.sunysb.edu

$\dagger$ Electronic address: flhalzen@facstaff.wisc.edu

${ }^{\ddagger}$ Electronic address: soumya@insti.physics.sunysb.edu
} 


\section{RESOLVING THE GALACTIC COSMIC RAY PROBLEM}

In 1934, Baade and Zwicky[1] pointed out that supernovae could be the sources of the Galactic cosmic rays provided that a substantial fraction of the energy released in the explosion is converted into the acceleration of relativistic particles. Their proposal has been commonly accepted despite the fact that to date no source has been conclusively identified. Because cosmic rays are deflected by magnetic fields, the sources must be identified by the accompanying pionic gamma rays and neutrinos produced when the accelerated particles interact with Galactic hydrogen.

Independently of the nature of the cosmic ray accelerator, it is a fact that galactic cosmic rays reach energies of at least several $\mathrm{PeV}$, the "knee" in the spectrum; their interactions should generate gamma rays and neutrinos from the decay of secondary pions reaching hundreds of $\mathrm{TeV}$. Such sources should be identifiable by a hard spectrum that extends to hundreds of $\mathrm{TeV}$ without attenuation; they have been dubbed PeVatrons. Indeed as we will emphasize in the next section, straightforward energetics arguments are sufficient to conclude that present air Cherenkov telescopes should have the sensitivity to detect $\mathrm{TeV}$ photons from PeVatrons. In fact, they may have been revealed by an all-sky survey in the $10 \mathrm{TeV}$ energy region with the Milagro detector[2]. A subset of sources located within nearby star forming regions in Cygnus and in the vicinity of galactic latitude $l=40$ degrees are identified that are not readily associated with known supernova remnants or with nonthermal sources observed at other wavelengths. Subsequently directional air Cherenkov telescopes were pointed at 3 of the sources revealing them as potential PeVatrons with an approximate $E^{-2}$ energy spectrum that extends to tens of $\mathrm{TeV}$ without evidence for a cutoff [3, 4]. This is in sharp contrast with the best studied supernova remnants RX J1713-3946 and RX J0852.0-4622 (Vela Junior).

The Milagro sources are suspected to be molecular clouds illuminated by the cosmic ray beam accelerated in young remnants located within $\sim 100$ pc. In brief, one expects that multi-PeV cosmic rays are accelerated only during a short period when the remnant transitions from the free-expansion to the beginning of the Sedov phase and the shock velocity is high. The high energy particles can produce photons and neutrinos over much longer times as they diffuse through the interstellar medium to interact with nearby molecular clouds;

for a detailed discussion see reference [5]. An association of molecular clouds and supernova 
remnants is expected in star forming regions.

Unfortunately the basic hurdle to conclusively associate the observed $\mathrm{TeV}$ gamma rays with the decay of pions produced by a cosmic accelerator has not been overcome. Synchrotron radiation by energetic electrons followed by inverse Compton scattering, routinely observed in non-thermal sources, cannot be excluded as their origin. Neutrinos from the decay of charged pions accompanying pionic gamma rays can provide incontrovertible evidence for cosmic ray acceleration in the source. It is the purpose of this paper to assess as realistically as possible the actual prospects for detecting them.

The outline of the paper is at follows. We first set the stage by selecting the $\mathrm{TeV}$ sources that can be reasonably expected to be candidate Galactic cosmic ray accelerators, i.e. supernova remnants or associated molecular clouds. We will subsequently derive the neutrino flux from data collected by TeV gamma ray detectors. Especially for the case of molecular clouds, the neutrino flux should be predictable at a quantitative level as any confusion of pionic gamma rays with gamma rays of electromagnetic origin should be minimal[5]. This argues for a more realistic evaluation of the potential of the IceCube detector taking fully account of the fact that the measurement of the energy of the secondary muons can be used to further discriminate between the signal and the background of atmospheric neutrinos.

Our results agree with previous estimates [6] that a neutrino signal should emerge after several years from the data of a kilometer-scale detector with the predicted fluxes at the level of the background. For average values of the parameters we find that the completed IceCube detector could confirm the sources in the Milagro sky map as sites of cosmic ray acceleration at the $3 \sigma$ level in less than one year and at the $5 \sigma$ level in three years. We discuss the considerable dependence of these expectations on the remaining ambiguities in this estimate. With the performance of IceCube relatively well understood, these are mostly of astrophysical origin. The absence of evidence after 10 years would definitely reopen the question of the origin of Galactic cosmic rays and represent an interesting challenge. Whereas other speculations for their origin exist, for instance micro-quasars, it is difficult to reconcile them with the fact that they do not appear in the Milagro sky.

Finally we briefly comment on the prospects for a northern detector such as KM3NeT viewing southern sources. Opportunities are actually limited; at this time there are only two candidate sources, RX J1713-3946 and Vela Junior. It is important to point out however that the northern sources viewed by IceCube were only revealed after the commissioning 
of all sky $\mathrm{TeV}$ instruments such as Milagro. No such instrument has studied the Southern hemisphere and, for the near future, the only opportunity to do so is to use IceCube as a gamma ray detector. A quantitative evaluation of this possibility is in progress[7].

\section{CANDIDATE GALACTIC ACCELERATORS}

The energy density of the cosmic rays in our Galaxy is $\rho_{E} \sim 10^{-12} \mathrm{erg} \mathrm{cm}^{-3}$. Galactic cosmic rays are not forever; they diffuse within the microgauss fields and remain trapped for an average containment time of $3 \times 10^{6}$ years. The power needed to maintain a steady energy density requires accelerators delivering $10^{41} \mathrm{erg} / \mathrm{s}$. This happens to be $10 \%$ of the power produced by supernovae releasing $10^{51}$ erg every 30 years $\left(10^{51} \mathrm{erg}\right.$ correspond to $1 \%$ of the binding energy of a neutron star after $99 \%$ is initially lost to neutrinos). This coincidence is the basis for the idea that shocks produced by supernovae exploding into the interstellar medium are the accelerators of the Galactic cosmic rays.

Despite the rapid development of instruments with improved sensitivity, it has been impossible to conclusively pinpoint supernova remnants as the sources of cosmic rays by identifying accompanying gamma rays of pion origin. A generic supernova remnant releasing an energy of $W \sim 10^{50} \mathrm{erg}$ into the acceleration of cosmic rays will inevitably generate $\mathrm{TeV}$ gamma rays by interacting with the hydrogen in the Galactic disk. The emissivity in pionic gamma rays $Q_{\gamma}$ is simply proportional to the density of cosmic rays $n_{c r}$ and to the target density $n$ of hydrogen atoms. Here $n_{c r} \simeq 4 \times 10^{-14} \mathrm{~cm}^{-3}$ is obtained by integrating the proton spectrum for energies in excess of $1 \mathrm{TeV}$. For a $E^{-2}$ spectrum,

$$
Q_{\gamma} \simeq c\left\langle\frac{E_{\pi}}{E_{p}}\right\rangle \lambda_{p p}^{-1} n_{c r}(>1 \mathrm{TeV}) \simeq 2 c x_{\gamma} \sigma_{p p} n n_{c r},
$$

or

$$
Q_{\gamma}(>1 \mathrm{TeV}) \simeq 10^{-29} \frac{\text { photons }}{\mathrm{cm}^{3} \mathrm{~s}}\left(\frac{n}{1 \mathrm{~cm}^{-3}}\right),
$$

The proportionality factor in Eq. (1) is determined by particle physics; $x_{\gamma}$ is the average energy of secondary photons relative to the cosmic ray protons and $\lambda_{p p}=\left(n \sigma_{p p}\right)^{-1}$ is the proton interaction length $\left(\sigma_{p p} \simeq 40 \mathrm{mb}\right)$ in a density $n$ of hydrogen atoms. The corresponding luminosity is

$$
L_{\gamma}(>1 \mathrm{TeV}) \simeq Q_{\gamma} \frac{W}{\rho_{E}}
$$


where $W / \rho_{E}$ is the volume occupied by the supernova remnant. We here made the approximation that the volume of the young remnant is approximately given by $W / \rho_{E}$ or, that the density of particles in the remnant is not very different from the ambient energy density $\rho_{E} \sim 10^{-12} \mathrm{erg} \mathrm{cm}^{-3}$ of Galactic cosmic rays.

We thus predict a rate of $\mathrm{TeV}$ photons from a supernova at a nominal distance $d$ of order $1 \mathrm{kpc}$ of

$$
\int_{E>1 \mathrm{TeV}} \frac{d N_{\gamma}}{d E_{\gamma}} d E_{\gamma}=\frac{L_{\gamma}(>1 \mathrm{TeV})}{4 \pi d^{2}} \simeq 10^{-12}-10^{-11}\left(\frac{\text { photons }}{\mathrm{cm}^{2} \mathrm{~s}}\right)\left(\frac{W}{10^{50} \mathrm{erg}}\right)\left(\frac{n}{1 \mathrm{~cm}^{-3}}\right)\left(\frac{d}{1 \mathrm{kpc}}\right)^{-2} .
$$

Such sources must emerge in an all-sky $\mathrm{TeV}$ gamma ray survey performed with an instrument with the sensitivity of the Milagro experiment [2].

Furthermore, as discussed in the introduction, the position of the knee in the cosmic ray spectrum indicates that some sources accelerate cosmic rays to energies of several $\mathrm{PeV}$. These PeVatrons therefore produce pionic gamma rays whose spectrum can extend to several hundred $\mathrm{TeV}$ without cutting off. For such sources the $\gamma$-ray flux in the $\mathrm{TeV}$ energy range can be parametrized in terms of a spectral slope $\alpha_{\gamma}$, an energy $E_{c u t, \gamma}$ where the accelerator cuts off and a normalization $k_{\gamma}$

$$
\frac{d N_{\gamma}\left(E_{\gamma}\right)}{d E_{\gamma}}=k_{\gamma}\left(\frac{E_{\gamma}}{\mathrm{TeV}}\right)^{-\alpha_{\gamma}} \exp \left(-\sqrt{\frac{E_{\gamma}}{E_{c u t, \gamma}}}\right)
$$

The estimate in Eq. (44) indicates that fluxes as large as $d N_{\gamma} / d E_{\gamma} \sim 10^{-12}-10^{-14}\left(\mathrm{TeV}^{-1}\right.$ $\left.\mathrm{cm}^{-2} \mathrm{~s}^{-1}\right)$ can be expected at energies of $\mathcal{O}(10 \mathrm{TeV})$.

To date, the Milagro collaboration has identified 6 such PeVatron candidates. Surprisingly, they cluster in star forming regions in the nearby spiral arms, four in the Cygnus region (MGRO J2019+37, MGRO J2031+41, MGRO J2043+36 and MGRO J2032+37) and two more (MGRO J1908+06 and MGRO J1852+01) near galactic longitude of $l=40$ degrees (where they are also within the field of view of the Southern telescopes).

The spectrum of 3 of the sources supports their identification as a PeVatron. H.E.S.S. observations of MGRO J1908+06 reveal a spectrum consistent with a $E^{-2}$ dependence from $500 \mathrm{GeV}$ to $40 \mathrm{TeV}$ without evidence for a cutoff [3]. In a follow-up analysis the MILAGRO collaboration [8] showed that its own data are consistent with an extension of the H.E.S.S. spectrum to at least $90 \mathrm{TeV}$. This is suggestive of pionic gamma rays from a PeVatron whose cosmic ray beam extends to the knee in the cosmic ray spectrum at PeV energies. MGRO 
J2031+41, has been observed [4] by the MAGIC telescope with a spectrum that is also consistent with $E^{-2}$. The lower flux measured by MAGIC, which we will conservatively adopt in our calculations, is likely attributed to the problem of differentiating the source from the background in a high density environment like the Cygnus region. Finally, the failure of Veritas to observe MGRO J2019+37 at lower energies implies that the slope of the spectrum must be larger than -2.2 .

In the end, despite the suggestive evidence, conclusively tracing the observed gamma rays to pions produced by cosmic-ray accelerators has so far been elusive. It is one of the main missions of neutrino telescopes to produce the smoking gun for cosmic-ray production by detecting neutrinos from charged pions. Neutrino telescopes detect the Cherenkov radiation from secondary particles produced by the interactions of high energy neutrinos in highly transparent and well shielded deep water or ice. They take advantage of the relatively large cross section of high-energy neutrinos and the long range of the muons produced. The first kilometer-scale detector, IceCube, is under construction at the geographic South Pole[9]. IceCube will consist of 80 kilometer-length strings, each instrumented with 60 10-inch photomultipliers spaced by $17 \mathrm{~m}$. The deepest module is located at a depth of $2.450 \mathrm{~km}$ so that the instrument is shielded from the large cosmic ray background at the surface by approximately $1.5 \mathrm{~km}$ of ice. The strings are arranged at the apexes of equilateral triangles $125 \mathrm{~m}$ on a side. The instrumented detector volume is a cubic kilometer of dark, transparent and sterile Antarctic ice. Each optical sensor consists of a glass sphere containing the photomultiplier and the electronics board that digitizes the signals locally using an onboard computer. The digitized signals are given a global time stamp with residuals accurate to less than 3 ns and are subsequently transmitted to the surface. Processors continuously collect the time-stamped signals from the optical modules that each function independently. The digital messages are sent to a string processor and a global event trigger. They are subsequently sorted into the Cherenkov patterns emitted by secondary muon tracks that reveal the direction of the parent neutrino[10]. Operating with 59 out of 80 strings, IceCube's total exposure will reach $1 \mathrm{~km}^{2}$ year in 2009. A more realistic evaluation of its potential to reveal galactic cosmic ray accelerators is therefore timely.

We list in table II the spectral parameters for the northern hemisphere sources selected as candidate sources. The choice of sources is somewhat arbitrary. We did include the relatively strong source MGRO J1852+01 despite the fact that it moved at some point below Milagro's 


\begin{tabular}{|l|c|c|c|l|}
\hline Source & $\frac{d N_{\gamma, i}\left(E_{\text {norm }}\right)}{d E_{\gamma}}\left(\mathrm{TeV}^{-1} \mathrm{~cm}^{-2} \mathrm{~s}^{-1}\right)$ & $E_{\text {norm }, i}(\mathrm{TeV})$ & $\alpha_{\gamma, i}$ & $E_{c u t, \gamma, i}(\mathrm{TeV})$ \\
\hline MGRO J2019+37 & $8.7 \times 10^{-15}$ & 20 & 2 & $25-800$ \\
MGRO J2031+41 & $1.7 \times 10^{-14}$ & 12 & 2 & $25-800$ \\
MGRO J2043+36 & $1.2 \times 10^{-14}$ & 12 & $1.5-3$ & $25-800$ \\
MGRO J2032+37 & $0.9 \times 10^{-14}$ & 12 & $1.5-3$ & $25-800$ \\
MGR0 J1908+06 & $8.8 \times 10^{-15}$ & 20 & 2 & $25-800$ \\
MGRO J1852+01 & $5.7 \times 10^{-14}$ & 12 & $1.5-3$ & $25-800$ \\
\hline
\end{tabular}

TABLE I: Normalization and parameters assumed for the six North Hemisphere potential PeVatrons considered in the text. The value of $k_{\gamma_{i}}$ for each source is obtained by imposing the normalization condition given in the second column of the table and are the same as in Ref.[11].

5 sigma pre-trial requirement. On the other hand, we did not include Geminga and MGRO J2226+60. The latter could actually be supernova remnant SNR G106.6+2.9; it could also be the Boomerang pulsar wind nebula. It is generally assumed that the TeV radiation from pulsar wind nebulae is electromagnetic in origin so we do not consider them as potential neutrino sources. One also has to realize that, after subtracting the sources considered, an excess of TeV gamma rays persists in the Milagro's skymap from the general direction of the Cygnus region [12]. This "diffuse" flux most likely originates in unresolved sources that contribute additional neutrinos.

The neutrino fluxes associated with pionic gamma rays emitted by a source is directly determined by particle physics; approximately one $\nu_{\mu}+\bar{\nu}_{\mu}$ pair should accompany every 2 gamma rays. The exact relation between the gamma ray and neutrino fluxes has been described in detail in Ref.[13]. Following their work we write the corresponding neutrino flux at the Earth after oscillations using the approximate relations [13, 14]

$$
\frac{d N_{\nu_{\mu}+\bar{\nu}_{\mu}, i}\left(E_{\nu}\right)}{d E_{\nu}}=k_{\nu, i}\left(\frac{E_{\nu}}{\mathrm{TeV}}\right)^{-\alpha_{\nu, i}} \exp \left(-\sqrt{\frac{E_{\nu}}{E_{c u t, \nu, i}}}\right)
$$

with

$$
\begin{aligned}
& k_{\nu}=\left(0.694-0.16 \alpha_{\gamma}\right) k_{\gamma} \\
& \alpha_{\nu}=\alpha_{\gamma} \\
& E_{c u t, \nu}=0.59 E_{c u t, \gamma}
\end{aligned}
$$

The predicted flux should be robust for the assumption tested that the sources are molecular 
clouds illuminated by the beam from nearby supernova remnants. Unlike what could be the case for the remnants themselves, a negligible[5] synchrotron component of the TeV radiation is expected that must be differentiated from the pionic gamma rays.

Thus the estimates of the neutrino flux are sufficiently quantitative and the ambiguities associated with the astrophysical parameters suitably defined, that a quantitative estimate of the response of IceCube is warranted at this point. Unlike what was done in previous estimates, we will simulate the detection at the level of the secondary muons. This allows us to study the influence of energy measurement which will turn out to be a powerful tool in rejecting the background of atmospheric neutrinos as we discuss next.

\section{RESULTS}

The number of events detected by IceCube from a source at zenith angle $\theta_{s}$ is

$$
\begin{gathered}
N_{e v}=t \times N_{T} \int d E_{\nu} d E_{\mu}^{0} d E_{\mu}^{f i n} \quad\left[\frac{d N_{\nu}\left(E_{\nu}\right)}{d E_{\nu}} \times A t t_{\nu}\left(E_{\nu}, \theta_{s}\right) \times \frac{d \sigma_{\nu}\left(E_{\nu}, E_{\mu}^{0}\right)}{d E_{\mu}^{0}}\right. \\
\left.+\frac{d N_{\bar{\nu}}\left(E_{\nu}\right)}{d E_{\nu}} \times A t t_{\bar{\nu}}\left(E_{\nu}, \theta_{s}\right) \times \frac{d \sigma_{\bar{\nu}}\left(E_{\nu}, E_{\mu}^{0}\right)}{d E_{\mu}^{0}}\right] \\
\times R R\left(E_{\mu}^{0}, E_{\mu}^{f i n}\right) \times A_{\mu}^{e f f}\left(E_{\mu}^{f i n}, \theta_{s}\right)
\end{gathered}
$$

The flux sums over equal numbers of neutrinos and antineutrinos of energy $E_{\nu}$ which are propagated through the Earth; their zenith-dependent attenuation is described by $\operatorname{Att}_{\nu}\left(E_{\nu}, \theta\right)$. The neutrinos subsequently interact with a cross section $\frac{d \sigma_{\nu}\left(E_{\nu}, E_{\mu}^{0}\right)}{d E_{\mu}^{0}}$ to yield a secondary muon of energy $E_{\mu}^{0}$. This muon reaches the detector with an energy $E_{\mu}^{f i n}$. The muon propagation is taken into account by $R R\left(E_{\mu}^{0}, E_{\mu}^{f i n}\right)$. The detector performance is described by its effective area for detecting muons $A_{\mu}^{\text {eff }}\left(E_{\mu}^{f i n}, \theta_{s}\right)$ and the exposure time $t$. $N_{T}$ is the target density of the material surrounding the detector. We emphasize that in order to make a realistic estimate of the sensitivity of a detector it is essential to describe its performance at the muon level because it is the muon, not the neutrino energy, that is measured. This has been overlooked in previous work. In order to perform the calculations we use the approach introduced in Ref.[15]; the details are collected in the appendix.

We subsequently calculate the background of atmospheric neutrinos at the zenith angle corresponding to the source using the Honda [16] flux which we extrapolate to higher energies to match the flux from Volkova [17]; the latter is known to describe the AMANDA data in the 
energy range of interest here. At high energy prompt neutrinos from charm decay contribute. In order to estimate the uncertainty associated with the calculation of the charm flux, we compute the expected number of events for two models of charm production that bracket predictions in the literature: the recombination quark parton model (RQPM) developed by Bugaev et al [18] and the model of Thunman et al (TIG) [19] that predicts a smaller rate. We integrate the atmospheric background over a solid angle $\Omega=\pi / 2(1.6 \delta \psi)^{2}$ around the direction of the source. The angle $\delta \psi$ combines the effects of the angular resolution of the detector and the size of the source. Assuming gaussianity, $70 \%$ of the flux of the source is contained within this angular bin.

We will bin the events in the measured muon energy $E_{\mu}^{f i n}$ assuming a given energy resolution above $E_{\mu}^{\text {fin }} \geq 1 \mathrm{TeV}$. Below $1 \mathrm{TeV}$ catastrophic energy loss is small and we assume no energy resolution; we sum these events in a unique bin.

Given that the event rates are expected to be a few per candidate source per year in IceCube, and therefore at the level of the background of atmospheric neutrinos, the usual procedure is to "stack" the sources within the field of view. As an illustration we show in Fig.1 the total number of events from the sum of the sources in Table II and the corresponding number of background events as a function of $E_{\mu}^{f i n}$. The results are shown for some representative choices of the angular bin size and assume an energy resolution of $30 \%$ in $\log \left(E_{\mu}^{f i n}\right)$ for $E_{\mu}^{f i n} \geq 1 \mathrm{TeV}$. The figure illustrates the dependence of signal rates on variations of the parameters describing the gamma ray flux were we have assumed the same $E_{c u t, \gamma}$ for all the sources. For the reasons discussed, we fix $\alpha_{\gamma}=2$ for MGRO J1908+06, MGRO J2031+41 and MGRO J2019+37 while for the other three sources, MGRO J2043+36, MGRO J2032+37, and MGRO J1852+01, we vary $\alpha_{\gamma}$ as shown in the figure. Where the choice of angular bin is concerned, while the angular resolution of IceCube is better than 1 degree for the effective area assumed, there is evidence that some sources extend over a radius of 1.5 degrees, hence our range of bin sizes considered.

Given an expected $E_{\mu}^{f i n}$ spectrum for the sources and for the background we simulate a large number of experiments that are Poisson distributed around the background, and around signal plus background. From these sets we obtain the significance or probability of the observation from the mean likelihood for the observed spectra - assumed to be that of the signal plus background - to be a random fluctuation of the background. We show in the upper panels of Fig 2 the expected significance of the IceCube observation as a function of 


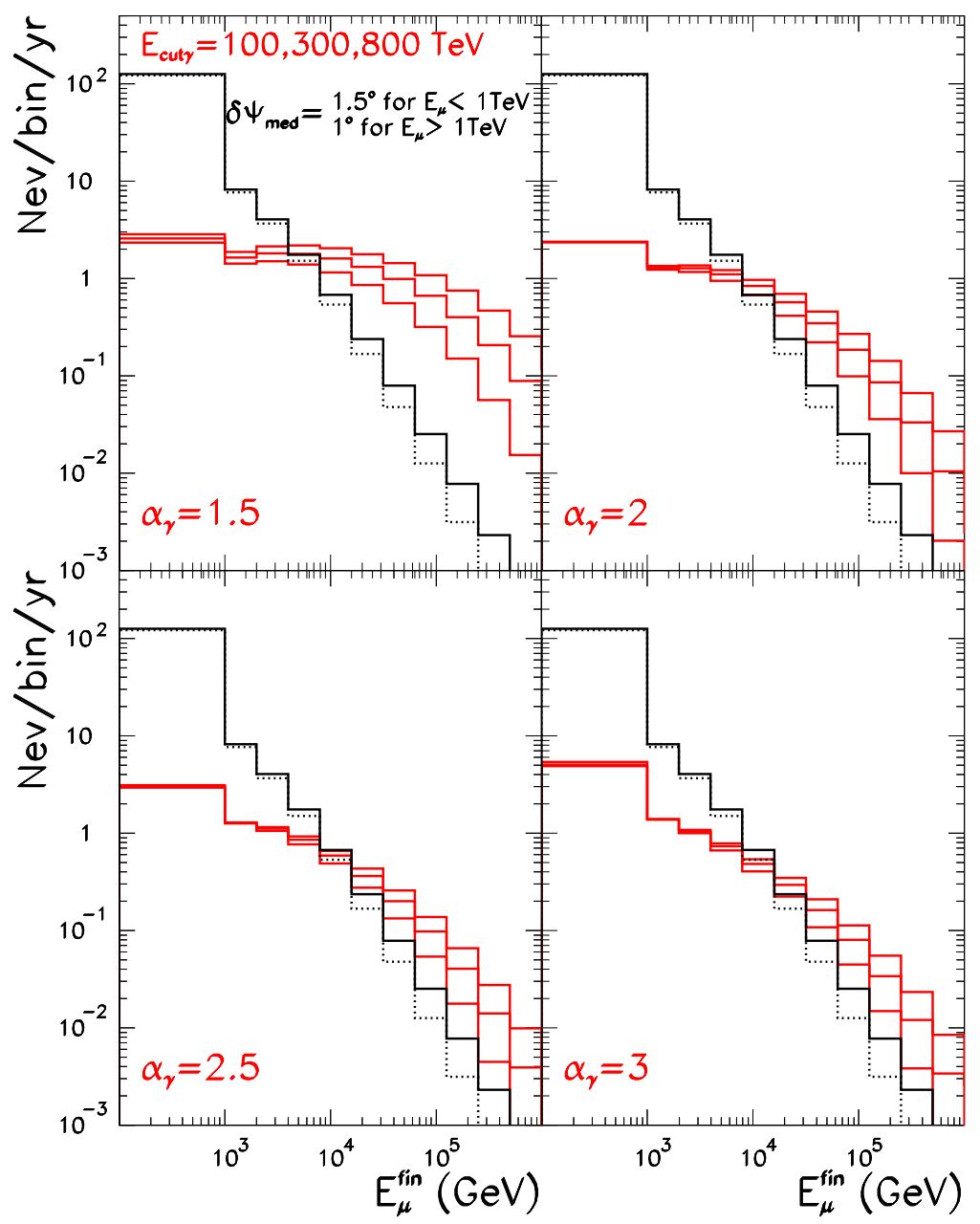

FIG. 1: Total number of events as a function of $E_{\mu}^{f i n}$ from the sum of all the sources (red histograms) and the corresponding total number of background event (steeper black histograms). Background is shown for the two models of charm considered: RQPM (full) and TIG (dotted). In each panel the higher, intermediate and lower curves for the expected number of events from the sources correspond to $E_{c u t, \gamma}=800,300$ and $100 \mathrm{TeV}$ respectively ( assumed to be the same for all the sources). In the different panels the $\alpha_{\gamma}$ for MGRO J2043+36, MGRO J2032+37 and MGRO $\mathrm{J} 1852+01$ is given in the figure, while in all cases we set $\alpha_{\gamma}=2$ for MGRO J1908+06, MGRO J2031+41 and MGRO J2019+37. In all cases, the background is integrated in an solid angle bin $\Omega=\pi / 2(1.6 \delta \psi)^{2}$ around the direction of the sources with $\delta \psi=1.5$ degrees for events with $E_{\mu}^{f i n}<1$ $\mathrm{TeV}$ and $\delta \psi=1$ degrees for events with $E_{\mu}^{f i n}>1 \mathrm{TeV}$. 
time for a range of angular bins and for source parameters $\alpha_{\gamma}=2 E_{c u t, \gamma}=300 \mathrm{TeV}$. The figure also shows the dependence of the significance on the energy resolution of the detector. From the figure we conclude that for these source parameters and for the nominal detector resolution of $30 \%$ in $\log \left(E_{\mu}\right)$ above $1 \mathrm{TeV}$ and for an angular bin of $\delta \psi=1.5$ (1) degrees for events with $E_{\mu}^{f i n}<1 \mathrm{TeV}\left(E_{\mu}^{f i n}>1 \mathrm{TeV}\right)$, the stacked source analysis yelds a significance of $3 \sigma(5 \sigma)$ after $\sim 1.5(4.5)$ years. Widening the opening of the angular bin by a factor 2 above $1 \mathrm{TeV}$ increases these times to $\sim 3.5$ and 11 years. This result is not very much affected by increasing or decreasing the energy resolution by a factor of 2 . The result does depend on the choice of sources previously discussed. For instance, if the relatively strong source MGRO J1852+01 were not included, the time for a $5 \sigma$ observation would increase by a factor $\sim 1.7-2$.

Conversely, in Fig. 3 , we show the dependence of the significance of the observations on the source parameters. The left panels in the figure show the values of $\alpha_{\gamma}$ and $E_{c u t, \gamma}$ for which the stacked source analysis leads to a significance of $\geq 5 \sigma$ in 5 or 10 years respectively. As in Fig,1 we have assume the same $E_{c u t, \gamma}$ for all the sources while we fix $\alpha_{\gamma}=2$ for MGRO J1908+06, MGRO J2031+41 and MGRO J2019+37.

Alternatively one can simulate experiments around the events expected from background and signal plus background for each source individually, and subsequently combine the mean likelihood for the observed spectra to be a random fluctuation of the background. Combining the six likelihoods one obtains the probability and significance of the search. This method leads to a higher significance since the expected background is similar for all sources but the expected number of events is clearly larger for some sources. In the previous approach the significance of the brighter sources is diluted. The results following this alternative statistical approach are shown in the lower panels of Fig, 2 and in the left panels of Fig 3 . For example from Fig, 2 we see that for the nominal detector resolution of $30 \%$ in $\log \left(E_{\mu}\right)$ above $1 \mathrm{TeV}$, and for an angular bin of $\delta \psi=1.5$ (1) degrees for events with $E_{\mu}^{f i n}<1 \mathrm{TeV}$ $\left(E_{\mu}^{f i n}>1 \mathrm{TeV}\right)$, the combined likelyhood analysis can yeld a significance of $3 \sigma(5 \sigma)$ after $\sim$ 1. (3) years.

We conclude that within uncertainties in the source parameters and the detector performance, confirmation that Milagro mapped the sources of the Galactic cosmic rays should emerge after operating the complete IceCube detector for several years. 


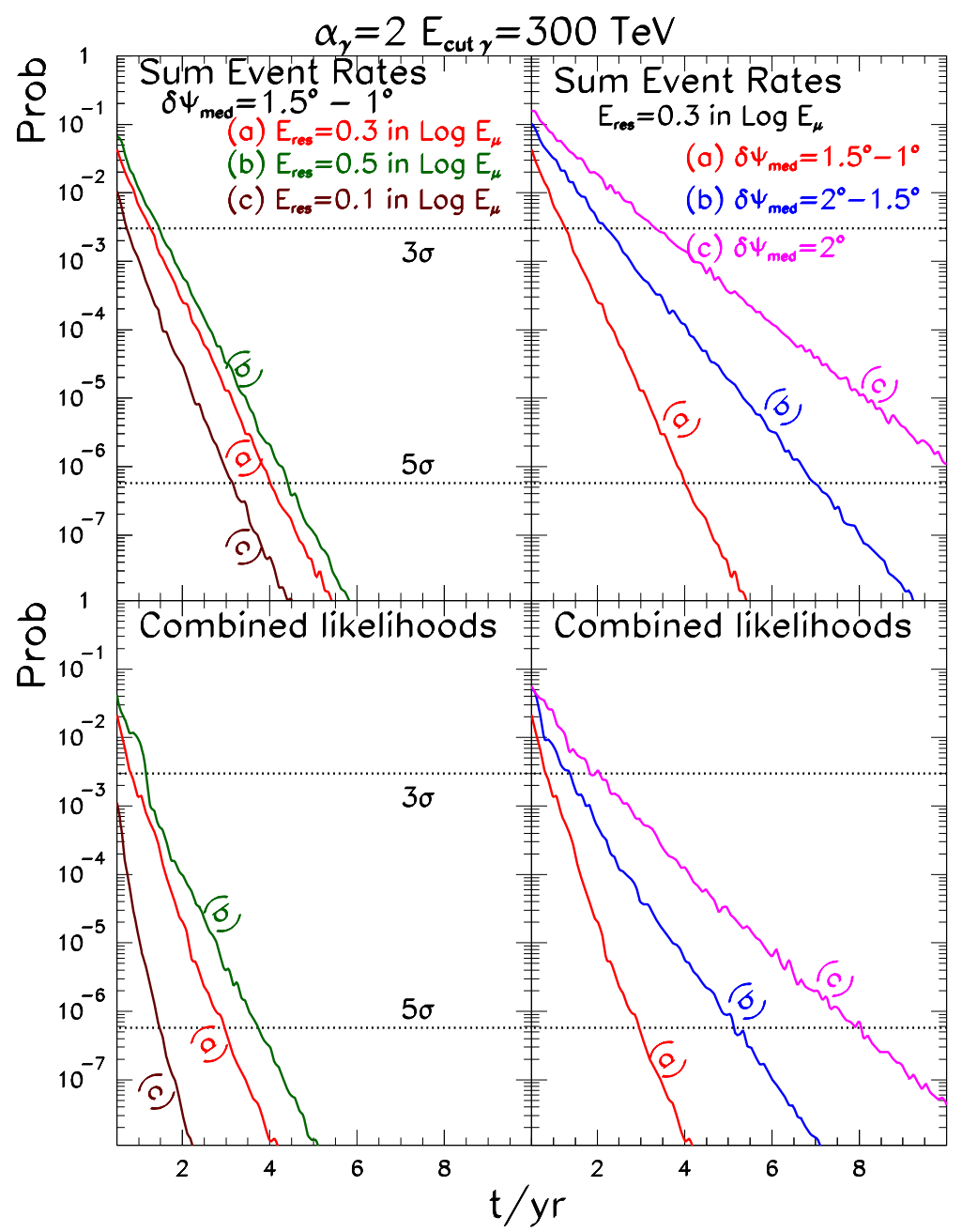

FIG. 2: Expected significance for the combined event rate analysis (upper panels) and for the combined likelihood analysis (lower panels) as a function of time for different angular bins (right panels) and energy resolution (left panels) assuming the same $\alpha_{\gamma}=2 E_{c u t, \gamma}=300 \mathrm{TeV}$ for the 6 sources. The charm contribution to the atmospheric background has been computed with the TIG model. In the left panels the background is integrated over $\Omega=\pi / 2(1.6 \delta \psi)^{2}$ with $\delta \psi=1.5(1)$ degrees for events with $E_{\mu}^{f i n}<1 \mathrm{TeV}\left(E_{\mu}^{f i n}>1 \mathrm{TeV}\right)$. The three curves correspond to energy resolution of (a) $30 \%$, (b) $50 \%$, and (c) $10 \%$ in $\log \left(E_{\mu}^{f i n}\right)$ for $E_{\mu}^{\text {fin }}>1 \mathrm{TeV}$. In the right panels an energy resolution of $30 \%$ in $\log \left(E_{\mu}^{f i n}\right)$ and the three curves correspond to background integrated over a solid angle bin $\Omega=\pi / 2(1.6 \delta \psi)^{2}$ around the direction of the sources with (a) $\delta \psi=1.5$ (1) degrees for events with $E_{\mu}^{f i n}<1 \mathrm{TeV}\left(E_{\mu}^{f i n}>1 \mathrm{TeV}\right)$, (b) $\delta \psi=2(1.5)$ degrees for events with $E_{\mu}^{f i n}<1.5 \mathrm{TeV}\left(E_{\mu}^{f i n}>1 \mathrm{TeV}\right)$, and (c) $\delta \psi=2$ degrees for all energies. 


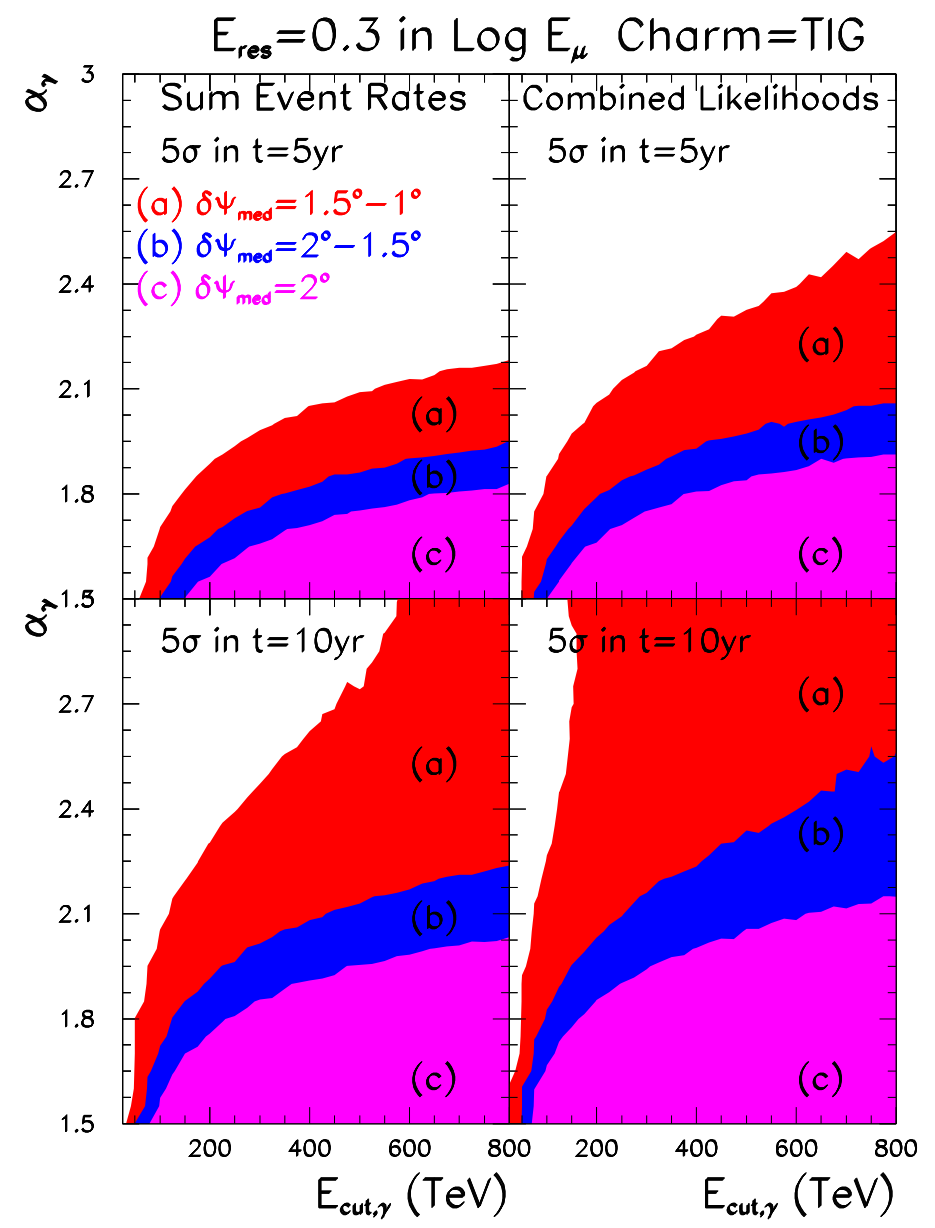

FIG. 3: Ranges of $\alpha_{\gamma}$ and $E_{c u t, \gamma}$ for which the combined event rate analysis of the Milagro sources leads to a significance of $\geq 5 \sigma$ in 5 (upper panels) and 10 (lower panels). years. In all panels we assume an energy resolution of $30 \%$ in $\log \left(E_{\mu}^{f i n}\right)$ and the three full regions (from outer to inner) correspond to background integrated over a solid angle bin $\Omega=\pi / 2(1.6 \delta \psi)^{2}$ around the direction of the sources with (a) $\delta \psi=1.5$ (1) degrees for events with $E_{\mu}^{f i n}<1 \mathrm{TeV}\left(E_{\mu}^{f i n}>1 \mathrm{TeV}\right)$, (b) $\delta \psi=2(1.5)$ degrees for events with $E_{\mu}^{f i n}<1.5 \mathrm{TeV}\left(E_{\mu}^{f i n}>1 \mathrm{TeV}\right)$, and (c) $\delta \psi=2$ degrees for all energies. We have assumed the same $E_{c u t, \gamma}$ for all the sources while we fix $\alpha_{\gamma}=2$ for MGRO J1908+06, MGRO J2031+41 and MGRO J2019+37. The left (right) panels corresponds to the analysis of the sum of the event rates (combined likelihoods) from the sources. 


\begin{tabular}{|l|c|c|c|}
\hline Source & $k_{\nu}\left(10^{-12} \mathrm{TeV}^{-1} \mathrm{~cm}^{-2} \mathrm{~s}^{-1}\right)$ & $\alpha_{\nu}$ & $E_{c u t, \nu}(\mathrm{TeV})$ \\
\hline RX J1713.7-3946 & 15.52 & 1.72 & 1.35 \\
RX J0852.0-4622 & 16.76 & 1.78 & 1.19 \\
Vela X & 11.75 & 0.98 & 0.84 \\
\hline
\end{tabular}

TABLE II: Normalization and parameters assumed for the three South Hemisphere sources considered in the text.

\section{A COMMENT ON OBSERVATIONS OF SOUTHERN HEMISPHERE SOURCES}

For comparison we have studied the expectations for a northern hemisphere detector, assumed to have a performance identical to IceCube, viewing supernova remnants in the southern hemisphere. We took the sources from Ref.[14]; the parameters describing their neutrino flux are compiled in Table【I. The event rates predicted are in agreement with those of Ref.[14] and our results shown in Fig,4. We show in Fig,5 the time dependence of the expected significance for the alternative ways of doing the statistics previously introduced.

Although the results are disappointing, this is most likely the result of insufficient information on southern PeVatrons. First, we omitted the Vela supernova remnant because it has not been detected in $\mathrm{TeV}$ gamma rays. Its pulsar, Vela X, is one of the strongest emitters of $\mathrm{TeV}$ radiation in the sky; the radiation is however believed to be of electromagnetic origin. Including it in the source list improves the results as seen in the dashed lines in the figure. It is possible that some of the unidentified H.E.S.S. sources should be included but in practice none of them contribute signals at the level of the three sources considered[14]. More importantly, before Milagro none of the sources studied in this paper had been detected in other wavelengths, or, if observed, had not been pinpointed as candidate PeVatrons. There have been no $\mathrm{TeV}$ all sky observations of the southern hemisphere. As previously pointed out, IceCube should attempt to identify such sources in the large background of cosmic ray muons which it collects at a rate of $1.5 \mathrm{KHz}$. 


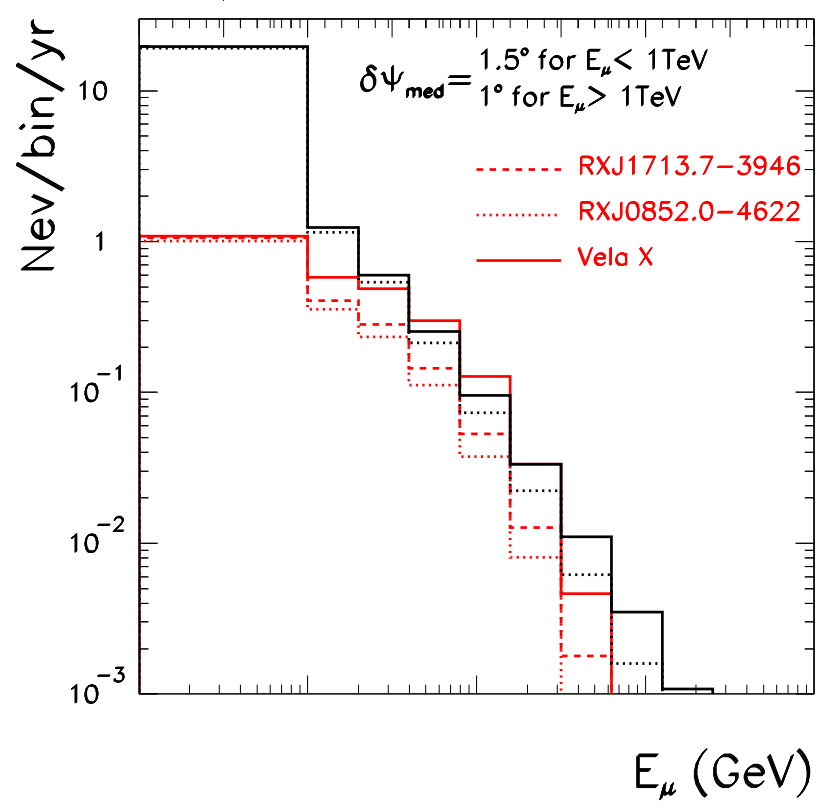

FIG. 4: Expected event rates from RX J1713.7-3946, RX J0852.0-4622, and Vela X as the atmospheric background for each source (black solid and dotted lines for the two models of charm considered).

\section{SUMMARY}

We have evaluated as realistically as possible the potential of IceCube to detect neutrinos in the direction of candidate sources for accelerators of the Galactic cosmic rays. In our calculation we make use of the secondary muon energy determination which allows to discriminate between the signal and the background from atmospheric neutrinos. In order to do so we have derived a quantitative description of the detector performance at the at the level of secondary muons in the form of a muon effective area.

Our results show that IceCube could identify the sources in the Milagro sky map as the sources of the Galactic cosmic rays at the $3 \sigma$ level in less than one year and at the $5 \sigma$ level in three years. We have studied the dependence of these expectations on the ambiguities associated with the astrophysical parameters characterizing the sources and with the detector performance. We conclude that the absence of evidence after ten years would reopen the question of the origin of Galactic cosmic rays. 


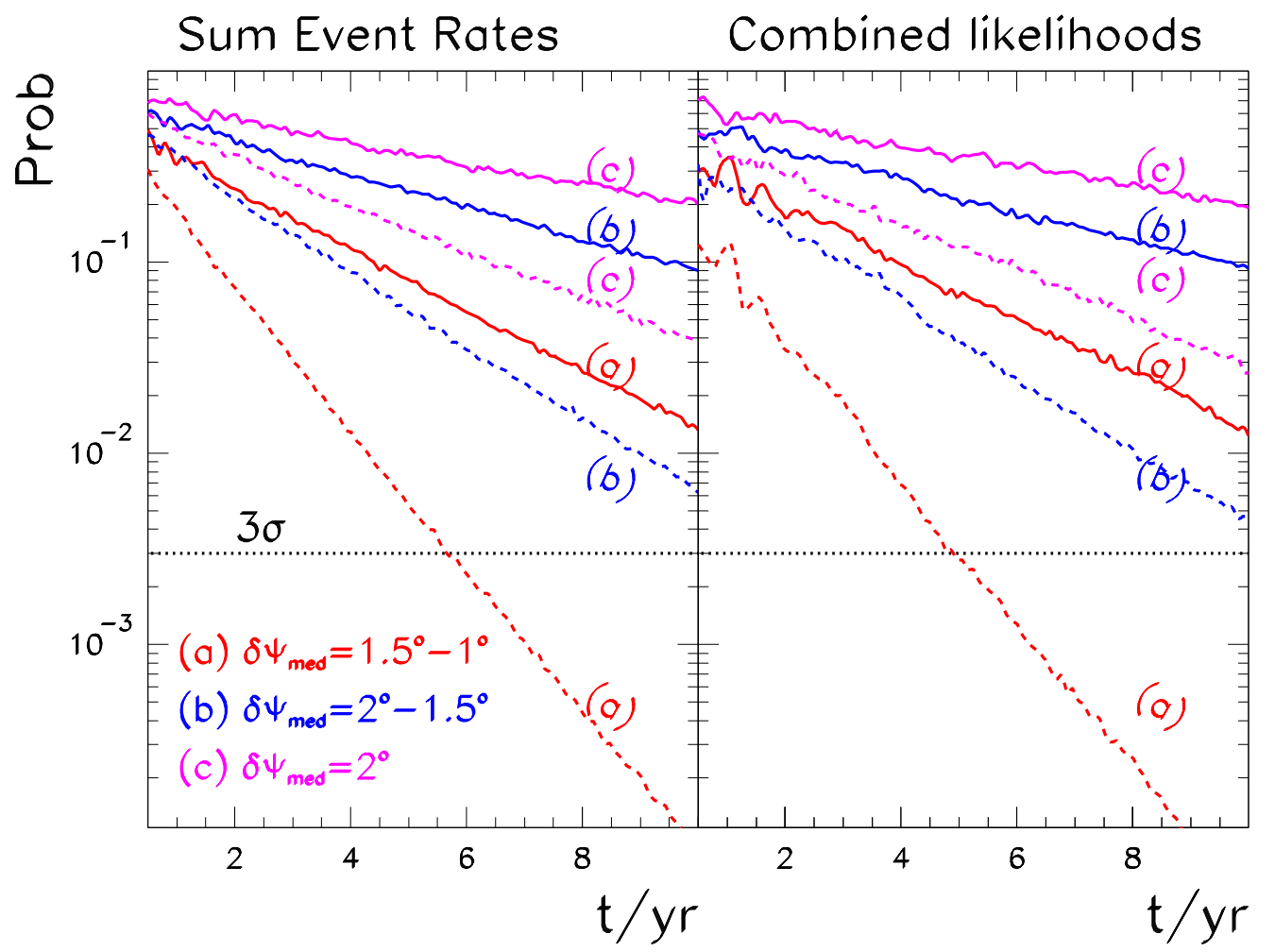

FIG. 5: Expected significance from the South Hemisphere sources RX J1713.7-3946 and RX J0852.0-4622 (full) and including also Vela X (dashed) as a function of time for different angular bins from the analysis of the summed event rates (left) and the combined likelihood functions (right). The charm contribution to the atmospheric background has been computed with the TIG model. In both panels we assume an energy resolution of $30 \%$ in $\log \left(E_{\mu}^{f i n}\right)$ and the colours correspond to background integrated over a solid angle bin $\Omega=\pi / 2(1.6 \delta \psi)^{2}$ around the direction of the sources with (a) $\delta \psi=1.5$ (1) degrees for events with $E_{\mu}^{f i n}<1 \mathrm{TeV}\left(E_{\mu}^{f i n}>1 \mathrm{TeV}\right)$, (b) $\delta \psi=2(1.5)$ degrees for events with $E_{\mu}^{f i n}<1.5 \mathrm{TeV}\left(E_{\mu}^{f i n}>1 \mathrm{TeV}\right)$, and (c) $\delta \psi=2$ degrees for all energies.

We briefly comment on the prospects for a northern detector such as KM3NeT viewing southern sources. They illustrate the potential importance of an all sky survey of the sourthern hemisphere. 


\section{Acknowledgments}

We thank Dmtri Chirkin and Darren Grant for discussions and contributions on the IceCube neutrino effective areas. We thank Alexander Kappes and Aongus O'Murchadha for comments and discussions. This research was supported by the National Science Foundation under Grants No. OPP-0236449 and PHY-0354776, by the U.S. Department of Energy under Grant No. DE-FG02-95ER40896, by the University of Wisconsin Research Committee with funds granted by the Wisconsin Alumni Research Foundation and by Spanish Grants FPA2007-66665-C02-01, FPA2006-28443-E and CSD2008-0037.

\section{APPENDIX: EFFECTIVE AREAS}

Our analysis illustrates the importance of describing the detection of neutrinos at the level of secondary muons in order to make a reliable estimate of the response of the detector to predictions. In this respect it is important to remark first that the neutrino energy is not measured by a neutrino telescope. The energy is that of the secondary muon when triggered by the instrument. The detector's performance is therefore described by the muon effective area $A_{\mu}\left(E_{\mu}, \theta\right)$. The number of observed events from a given neutrino flux is obtained by convoluting the propagated neutrino flux with the differential neutrino cross section, the muon propagation and the muon effective area.

At present the IceCube collaboration exclusively defines the performance of the detector by the neutrino effective area that simply yields the number of observed events after convolution with the neutrino flux. This makes the calculation of the total event rates simple but does not allow for a realistic evaluation of the significance of signals which relies on energy measurement. Furthermore, the neutrino effective area is not unique as it depends on the ratio of neutrino and antineutrino fluxes. It is subject to theoretical ambiguities associated with the neutrino cross section and muon range. We remark that all these ambiguities are independent of the details of the detector performance.

We here describe how we construct a muon area from the neutrino area ${ }^{1}$. It is important to point out that the muon area quantitatively reproduces identical event rates to the neutrino area at all energies. It is however a theoretical construct only because we have to make a

\footnotetext{
1 The issue has also been recently revisited in Ref. [21].
} 
working identification of the experimentally measured muon energy and a rather arbitrary variable describing the same quantity in our calculations. Following reference [15], we have made the identification $E_{\mu}^{\text {det }}=E_{\mu}^{\text {fin }}$. In doing so we rely on a procedure that was validated with the last muon area published by the collaboration [15, 22].

We start with the total (i.e. angular integrated) neutrino and antineutrino effective areas of Icecube [20], $A_{\nu}^{E f f}\left(E_{\nu}, \operatorname{bin}\right)$ and $A_{\bar{\nu}}^{E f f}\left(E_{\nu}\right.$, bin $)$, given in angular bins labeled $\operatorname{bin} \theta$, where $\theta$ is the zenith angle measured from the vertical. We obtain the corresponding muon effective area in the same angular bin, $A_{\mu}^{E f f}\left(E_{\mu}^{f i n}, \operatorname{bin} \theta\right)$, from the requirement that both should lead to the same number of events for an arbitrary flux. For illustration we plot in Fig [6 the average of neutrino plus antineutrino effective area averaged also over the northern hemisphere from Icecube [20]

$$
A_{\nu+\bar{\nu}}^{E f, a v}\left(E_{\nu}\right)=\frac{1}{2 \pi} \sum_{b i n} \frac{1}{2}\left(A_{\nu}^{E f f}\left(E_{\nu}, \operatorname{bin} \theta\right)+A_{\bar{\nu}}^{E f f}\left(E_{\nu}, \operatorname{bin} \theta\right)\right) .
$$

In terms of the neutrino area the number of events per unit time is given by

$$
\frac{N_{e v}}{d t}=\sum_{b i n} \int d E_{\nu}\left(\frac{d N_{\nu}\left(E_{\nu}, \operatorname{bin} \theta\right)}{d E_{\nu}} \times A_{\nu}^{E f f}\left(E_{\nu}, \operatorname{bin} \theta\right)+\frac{d N_{\bar{\nu}}\left(E_{\nu}, \operatorname{bin} \theta\right)}{d E_{\nu}} \times A_{\bar{\nu}}^{E f f}\left(E_{\nu}, \operatorname{bin} \theta\right)\right),
$$

where $d N_{\nu(\bar{\nu})}\left(E_{\nu}, \operatorname{bin} \theta\right) / d E_{\nu}$ is the angular-averaged, energy-differential neutrino (antineutrino) flux in the zenith angle bin $\operatorname{bin} \theta$.

Equivalently the number of events can be obtained in terms of the muon effective area as:

$$
\begin{gathered}
\frac{d N_{e v}}{d t}=N_{T} \sum_{b i n} \int d E_{\nu} d E_{\mu}^{0} d E_{\mu}^{f i n}\left[\frac{d N_{\nu}\left(E_{\nu}, \operatorname{bin} \theta\right)}{d E_{\nu}} \times A t t_{\nu}\left(E_{\nu}, \operatorname{bin} \theta\right) \times \frac{d \sigma_{\nu}\left(E_{\nu}, E_{\mu}^{0}\right)}{d E_{\mu}^{0}}\right. \\
\left.+\frac{d N_{\bar{\nu}}\left(E_{\nu}, b i n \theta\right)}{d E_{\nu}} \times A t t_{\bar{\nu}}\left(E_{\nu}, \operatorname{bin} \theta\right) \times \frac{d \sigma_{\bar{\nu}}\left(E_{\nu}, E_{\mu}^{0}\right)}{d E_{\mu}^{0}}\right] \\
\times R R\left(E_{\mu}^{0}, E_{\mu}^{f i n}\right) \times A_{\mu}^{\text {eff }}\left(E_{\mu}^{f i n}, \operatorname{bin} \theta\right) .
\end{gathered}
$$

In Eq. (11) $E_{\mu}^{0}$ labels the muon energy at its production point and $E_{\mu}^{f i n}$ its energy when it is detected after ranging out in the rock and the ice surrounding the detector. $N_{T}$ is the number density of targets in the vicinity of the detector. $d \sigma_{\nu}\left(E_{\nu}, E_{\mu}^{0}\right) / d E_{\mu}^{0}$ is the differential deep inelastic cross section for which we use the full expression (without any average inelasticity approximation) obtained with the CTEQ5 PDFs [23]. $\operatorname{Att}_{\nu(\bar{\nu})}\left(E_{\nu}, \operatorname{bin} \theta\right)$ is a factor which accounts for the attenuation of the flux due to neutrino (antineutrino) propagation in the 
Earth. It is consistently described by a set of coupled partial integro-differential cascade equations (see for example [15, 24] and references therein). The result can be approximated by [25, 26]

$$
\operatorname{Att}_{\nu(\bar{\nu})}\left(E_{\nu}, \operatorname{bin} \theta\right)=\exp \left[-X(\theta)\left(\sigma_{\mathrm{NC}}(E)+\sigma_{\mathrm{CC}}(E)\right)\right]
$$

where $X(\theta)$ is the column density of the Earth assuming the matter density profile of the Preliminary Reference Earth Model [27].

$R R\left(E_{\mu}^{0}, E_{\mu}^{f i n}\right)$ is the factor which describes the muon propagation from production to detection:

$$
R R\left(E_{\mu}^{0}, E_{\mu}^{f i n}\right)=\int_{0}^{\infty} F\left(E_{\mu}^{0}, E_{\mu}^{f i n}, l\right) d l,
$$

where we denote by $F\left(E_{\mu}^{0}, E_{\mu}^{\mathrm{fin}}, l\right)$ the probability that a muon produced with energy $E_{\mu}^{0}$ arrives at the detector with energy $E_{\mu}^{\text {fin }}$ after traveling a distance $l$. We compute the function $F\left(E_{\mu}^{0}, E_{\mu}^{\text {fin }}, l\right)$ by propagating the muons to the detector taking into account energy losses due to ionization, bremsstrahlung, $e^{+} e^{-}$pair production and nuclear interactions according to Ref. [26]. In particular we include in $F\left(E_{\mu}^{0}, E_{\mu}^{\mathrm{fin}}, l\right)$ the possibility of fluctuations around the average muon energy loss (using the average energy loss would identify $l$ with the average muon range distance). Thus in our calculation we keep $E_{\mu}^{0}, E_{\mu}^{\mathrm{fin}}$, and, $l$ as independent variables. Technically, this is done by numerically solving the one-dimensional integro-differential equation describing the muon propagation in matter (see, for example the appendix A of Ref. [26] and references therein).

Comparing Eq.(10) and Eq(11) we find the relation between the muon and the neutrino areas:

$$
\begin{aligned}
A_{\nu}^{E f f}\left(E_{\nu}, \operatorname{bin} \theta\right)= & N_{T} \int d E_{\mu}^{0} d E_{\mu}^{f i n} A t t_{\nu}\left(E_{\nu}, \operatorname{bin} \theta\right) \times \frac{d \sigma_{\nu}\left(E_{\nu}, E_{\mu}^{0}\right)}{d E_{\mu}^{0}} \\
& \times R R\left(E_{\mu}^{0}, E_{\mu}^{f i n}\right) \times A_{\mu}^{e f f}\left(E_{\mu}^{f i n}, \operatorname{bin} \theta\right),
\end{aligned}
$$

and equivalently for $A_{\bar{\nu}}^{E f f}\left(E_{\nu}, \operatorname{bin} \theta\right)$.

It is clear from Eq.(14) that without any further approximation $A_{\mu}^{e f f}\left(E_{\mu}^{f i n}, \operatorname{bin} \theta\right)$ cannot be directly computed from the neutrino effective areas because they are a double convolution of the muon areas. In order to extract the $A_{\mu}^{e f f}\left(E_{\mu}^{f i n}, \operatorname{bin} \theta\right)$ we assume some parametrization for its dependence on $E_{\mu}^{f i n}$ and $\theta$ and adjust the parameters to better describe the neutrino areas. 


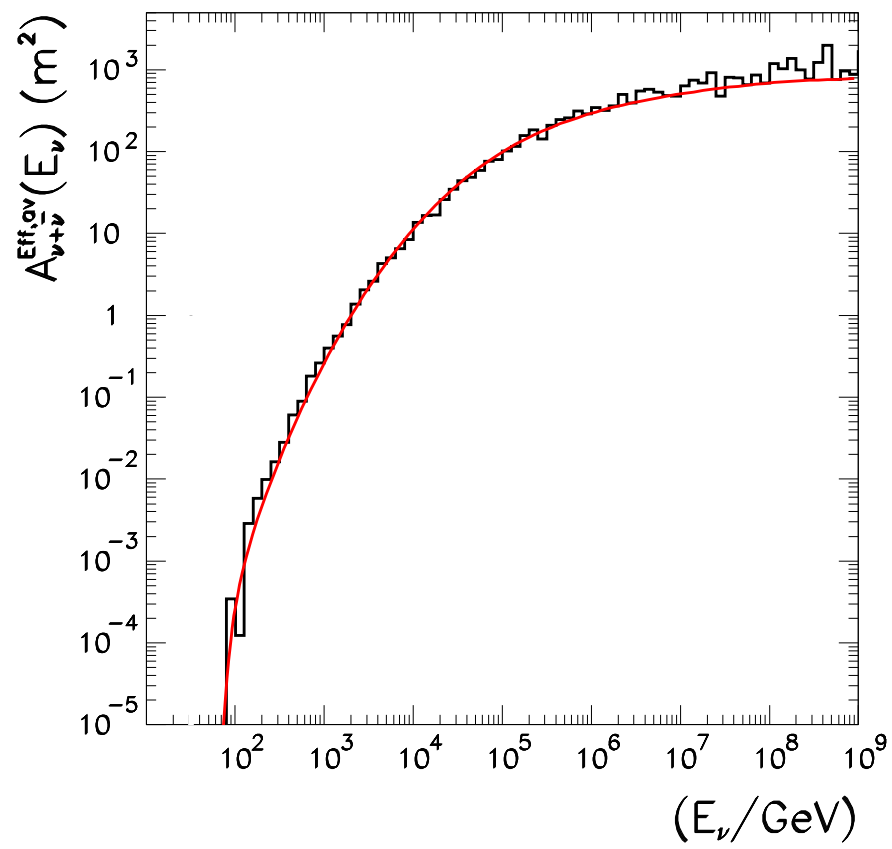

FIG. 6: The neutrino effective area (averaged over the north hemisphere) from the Icecube MCs (black histograms) is compared to the convolution of our fitted muon effective area (full red line).

Overall we find good agreement with a very simple functional form:

$$
A_{\mu}^{e f f}\left(E_{\mu}^{f i n}, \operatorname{bin} \theta\right)=2 \pi \int_{b i n \theta} A\left(E_{\mu}^{f i n}\right) \times R(\cos \theta) d \cos \theta
$$

with

$$
\begin{aligned}
& A\left(E_{\mu}^{\text {fin }} \leq 10^{1.6} \mathrm{GeV}\right)=0 \\
& A\left(10^{1.6} \mathrm{GeV} \leq E_{\mu}^{\text {fin }} \leq 10^{2.8} \mathrm{GeV}\right)=0.748\left[\log \left(E_{\mu}^{\text {fin }} / \mathrm{GeV}\right)-1.6\right] \\
& \left.A\left(10^{2.8} \mathrm{GeV}\right) \leq E_{\mu}^{\text {fin }}\right)=0.9+0.54\left[\log \left(E_{\mu}^{\text {fin }} / \mathrm{GeV}\right)-2.8\right]
\end{aligned}
$$

and

$$
R(\cos \theta)=0.92-0.45 \cos \theta
$$

We show in Fig, 6 the comparison between the Icecube effective area and the one which is obtained from the convolution of the muon effective area in Eqs. (15-17). 
[1] W. Baade and F. Zwicky, Phys. Rev. D 46, 76 (1934).

[2] A. A. Abdo et al., Astrophys. J. 658 L33-L36 (2007) arXiv:astro-ph/0611691.

[3] A. Djannati-Atai et al. [H.E.S.S. Collaboration], 30th ICRC, Merida, Mexico, 2007, arXiv:0710.2418.

[4] J. Albert et al., arXiv/astro-ph 0801.2391.

[5] For a recent discussion; see S. Gabici, F. .A. Aharonian, arXiv:0705.3011.

[6] L. Anchordoqui, F. Halzen, T. Montaruli and A. O'Murchadha, Phys. Rev. D 76, 067301 (2007), arXiv:astro-ph/0612699; J. F. Beacom and M. D. Kistler, Phys. Rev. D 75083001 (2007), arXiv:astro-ph/0701751; F. Halzen and A. O’Murchadha, Phys. Rev. D 76, 123003 (2007), arXiv:astro-ph/0705.1723.

[7] F. Halzen, A. Kappes and A. O’Murchadha (in preparation).

[8] S. Valenti [Milagro Collaboration], Non-thermal Hadronic Processes in Galactic Sources Workshop, Heidelberg, Germany, 2008.

[9] S. R. Klein [IceCube Collaboration], arXiv:0807.0034 [physics.ins-det].

[10] F. Halzen, Eur. Phys. J. C 46, 669 (2006) arXiv:astro-ph/0602132.

[11] F. Halzen, A. Kappes and A. O’Murchadha, Phys. Rev. D 78, 063004 (2008) arXiv:0803.0314 [astro-ph]].

[12] A. A. Abdo et al., arXiv:0805.0417 [astro-ph].

[13] S. R. Kelner, F. A. Aharonian and V. V. Bugayov, "Energy spectra of gamma-rays, electrons and neutrinos produced at proton Phys. Rev. D 74, 034018 (2006) arXiv:astro-ph/0606058.

[14] A. Kappes, J. Hinton, C. Stegmann and F. A. Aharonian, Astrophys. J. 656, 870 (2007) arXiv:astro-ph/0607286].

[15] M. C. Gonzalez-Garcia, F. Halzen and M. Maltoni, Phys. Rev. D 71, 093010 (2005) arXiv:hep-ph/0502223.

[16] M. Honda, T. Kajita, K. Kasahara and S. Midorikawa, Phys. Rev. D 70, 043008 (2004).

[17] L. V. Volkova, Sov. J. Nucl. Phys. 31, 784 (1980) [Yad. Fiz. 31, 1510 (1980)].

[18] E. V. Bugaev, A. Misaki, V. A. Naumov, T. S. Sinegovskaya, S. I. Sinegovsky and N. Takahashi, Phys. Rev. D 58, 054001 (1998)

[19] P. Gondolo, G. Ingelman and M. Thunman, Astropart. Phys. 5, 309 (1996). 
[20] A. Achterberg et al. [IceCube Collaboration], Astropart. Phys. 26, 155 (2006), arXiv:astro-ph/0604450; T. Montaruli et al. [IceCube Collaboration], in Proc. of Topics in Astroparticle and Underground Physics (TAUP07), Sendai, Japan, 2007. The neutrino effective area has been updated, see D. Grant, Novel Searches for Dark Matter with Neutrino Telescopes, Ohio State (2008) and D. Chirkin (private communication).

[21] A. Neronov and M. Ribordy, arXiv:0812.0306 [astro-ph].

[22] J. Ahrens et al. [IceCube Collaboration], Astropart. Phys. 20, 507 (2004) arXiv:astro-ph/0305196].

[23] H. L. Lai et al. [CTEQ Collaboration], Eur. Phys. J. C 12, 375 (2000).

[24] S. I. Dutta, M. H. Reno, I. Sarcevic and D. Seckel, Phys. Rev. D 63, 094020 (2001); S. I. Dutta, M. H. Reno and I. Sarcevic, Phys. Rev. D 66, 077302 (2002); J. Jones, I. Mocioiu, M. H. Reno and I. Sarcevic, Phys. Rev. D 69, 033004 (2004); S. Iyer, M. H. Reno and I. Sarcevic, Phys. Rev. D 61, 053003 (2000).

[25] T. K. Gaisser, Cosmic Rays And Particle Physics, Cambridge, UK: Univ. Pr. (1990).

[26] P. Lipari and T. Stanev, Phys. Rev. D 44 (1991) 3543.

[27] A.M. Dziewonski, D.L. Anderson, Phys. Earth Planet. Inter. 25, 297 (1981). 